\title{
Recent Advances in the Molecular Imaging of Programmed Cell Death: Part II-Non-Probe-Based MRI, Ultrasound, and Optical Clinical Imaging Techniques
}

\author{
Francis G. Blankenberg ${ }^{1}$ and H. William Strauss ${ }^{2}$ \\ ${ }^{I}$ Division of Pediatric Radiology, Department of Radiology, Lucile Salter Packard Children's Hospital, Stanford, California; \\ and ${ }^{2}$ Nuclear Medicine Service, Department of Radiology, Memorial Sloan-Kettering Cancer Center, New York, New York
}

\begin{abstract}
There is much that can be done to detect apoptosis and other forms of cell death with existing clinical modalities including ultrasound, MRI, and optical imaging without the need for current or new intravenous contrast agents. We will discuss how these widely available imaging technologies can readily be applied to the imaging of apoptosis in patients undergoing chemotherapy or radiation treatment. The limiting factor of course is the lack of knowledge of the optimal times after the start of treatment for the most accurate assessment of apoptosis and necrosis with each modality and specific technique. It is hoped that imaging studies that systematically look at treatment response can soon be performed to address these issues.
\end{abstract}

Key Words: apoptosis; necrosis; ultrasound; MRI; MRS; optical imaging

J Nucl Med 2013; 54:1-4

DOI: 10.2967/jnumed.112.111740

In this companion article to "Part I-Pathophysiology and Radiotracers" (1), we will now shift our focus to the review of existing nonradionuclide methods to image apoptosis in vivo. As outlined in the previous review, there are several stereotypical morphologic changes of the cell membrane, cytoplasm, and nucleus that can readily be detected by existing imaging technologies, including proton magnetic resonance spectroscopy ( $\left.{ }^{1} \mathrm{H}-\mathrm{MRS}\right)$, diffusionweighted MRI (DWI), high-frequency ultrasound, and optical imaging techniques without the use of intravenous contrast agents. We will briefly review each of these clinically available modalities and how, even without the use of intravenous contrast agents, they can be applied to the imaging of apoptosis.

\section{MRI TECHNIQUES FOR IMAGING APOPTOSIS}

${ }^{1} \mathrm{H}-\mathrm{MRS}$ and DWI take advantage of the apoptosisrelated biochemical, molecular diffusion, and morphologic

Received Jul. 25, 2012; revision accepted Oct. 1, 2012.

For correspondence or reprints contact: Francis G. Blankenberg, Stanford/ Lucile Packard Children's Hospital, 725 Welch Rd., Palo Alto, CA 94304.

E-mail: blankenb@stanford.edu

Published online Nov. 15, 2012.

COPYRIGHT (C) 2013 by the Society of Nuclear Medicine and Molecular Imaging, Inc. cellular changes summed over the bulk of a target such as a tumor or specific organ (i.e., brain or liver). Although the sensitivity of these MRI techniques is increased by looking at the behavior of a target, organ tissue, or tumor as a whole, there is a tradeoff with decreased spatial resolution. This limits MRI sequences to the study of apoptosis that affects a large portion or the whole of a target tumor or region of interest for a sufficient specific signal relative to noise. MRI sequences are also susceptible to motion artifacts and the bleeding-in of subcutaneous-fat signals or tissue-air magnetic inhomogeneities. Despite these limitations, MRI techniques still have great potential for measuring apoptosis in the brain, liver, and solid tumors.

\section{Assessment of Mobile Lipids and Cytoplasmic Lipid Droplets by ${ }^{\mathbf{1}} \mathrm{H}-\mathrm{MRS}$}

An early change in apoptosis is an increase in plasma membrane fluidity (without a change in lipid composition) as shown in Figure 1. Investigators found that in cell cultures, ${ }^{1} \mathrm{H}-\mathrm{MRS}$ was able to detect a specific increase in $-\mathrm{CH}_{2}-$ (methylene) relative to $-\mathrm{CH}_{3}$ (methyl) mobile lipid proton signal intensities at 1.3 and $0.9 \mathrm{ppm}$, respectively (2-4). The rise in $-\mathrm{CH}_{2}$ - resonance occurred when cells were treated with proapoptotic drugs or serum (growth factor) deprivation. In contrast, necrosis was characterized by a completely different ${ }^{1} \mathrm{H}$-MRS profile in which there was a significant increase in the resonance signal of most of the metabolites examined, with the exception of $\mathrm{CH}_{2}$ mobile lipids, which remain unchanged (coupled to a decrease in reduced glutathione). The ratio of $\mathrm{CH}_{2} / \mathrm{CH}_{3}$ signal intensity demonstrated a strong linear and temporal correlation with other markers of apoptosis, including fluorescent annexin V cytometry and DNA ladder formation.

Subsequent studies have shown that mobile lipid resonances can also arise from the osmophilic lipid droplets $(0.2-2.0 \mu \mathrm{m})$ observed in the cytoplasm in some models of apoptosis (5). These droplets contain variable amounts of polyunsaturated fatty acids associated largely with 18:1 and 18:2 lipid moieties (2.8 and $5.4 \mathrm{ppm}$ ) produced by both normal and oxidative metabolism. The droplets are also associated with an accumulation of triacylglycerides generated by apoptosis-induced phospholipase- $\mathrm{A}_{2}$ activity and 


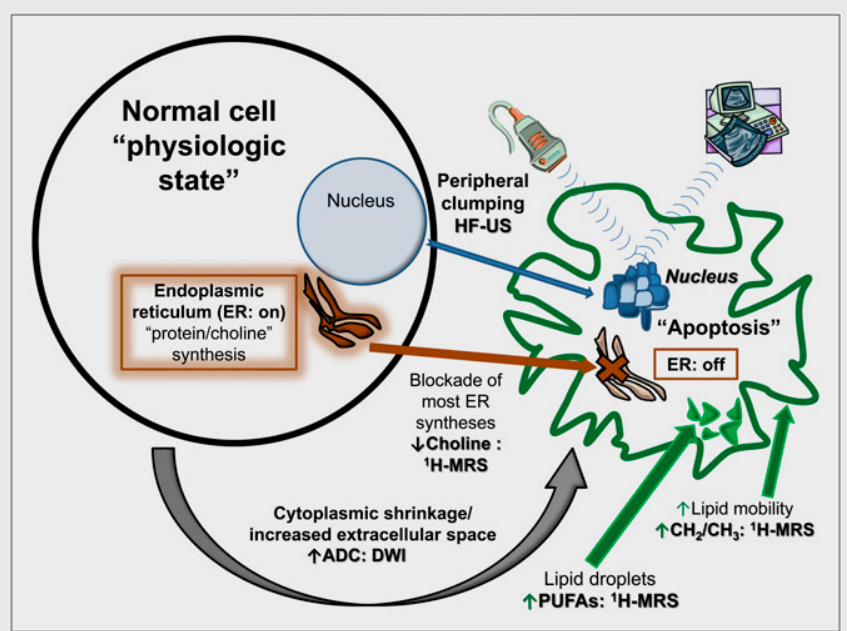

FIGURE 1. Non-probe-based modalities for imaging of apoptosis. Physiologically normal cell is shown on left. Arrows show various pathways that are activated or deactivated with apoptosis. Resultant effects on cell in apoptotic state are shown on right. Normal nucleus (blue) and its fragmentation into clumps of autodigested DNA and chromatin with apoptosis are illustrated. Fragmented nuclear material causes increase in backscatter that can readily be detected by high-frequency ultrasound (HF-US). Endoplasmic reticulum (ER, brown) is shown in cell in normal protein-synthesizing state (ER: on) and in apoptotic cell (ER: off) that effectively shuts choline synthesis off and is visible on ${ }^{1} \mathrm{H}$ MRS. Shrinkage and blebbing of plasma membrane and formation of lipid droplets containing neutral lipids, triglycerides, cholesterol, and increased polyunsaturated fatty acids (PUFAs) with apoptosis are shown in green. ${ }^{1} \mathrm{H}-\mathrm{MRS}$ can detect increases in lipid mobility (methylene/methyl lipid protons $=\mathrm{CH}_{2} / \mathrm{CH}_{3}=1.3 / 0.9 \mathrm{ppm}$ ) and PUFAs (seen at 2.8 and 5.4 ppm). Shrinkage of cell and increased extracellular space is indicated by gray arrow (ADC = increases in average diffusion coefficient with apoptosis that can be seen with DWI).

ceramide released by the hydrolysis of sphingomyelin by the membrane enzyme, sphingomyelinase. Because lipid droplets can be seen in both apoptosis and necrosis, the specificity of the signals observed at 2.8 and $5.4 \mathrm{ppm}$ is a potential problem (4).

More recent studies have suggested normalization of the $-\mathrm{CH}_{2}$ - resonance to the average intensity of a broad region of the spectrum from 1.6 to $4.7 \mathrm{ppm}(6)$. Using this normalization approach, it is possible to detect 4- to 5-fold increases in - $\mathrm{CH}_{2}$ - signal in breast carcinoma cell lines undergoing apoptosis in response to docetaxel without interference from the liposomes used to deliver the drug in culture. ${ }^{1} \mathrm{H}$-MRS measurements of radiation-induced apoptosis of cervical carcinoma are also possible with clinical MRI units using standard endovaginal coils $(7,8)$.

\section{Assessment of Total Choline by ${ }^{1} \mathrm{H}$-MRS}

${ }^{1} \mathrm{H}-\mathrm{MRS}$ can also detect significant decreases in the choline (including choline, choline-containing compounds, and phospholipids) resonance $6-12 \mathrm{~h}$ after the rise in mobile lipid signal in vitro (9). Lindskog originally proposed leveraging of the polar-opposite changes in mobile lipid and choline seen with apoptosis by calculating the ratio of $-\mathrm{CH}_{2}-$ signal intensity to choline signal intensity for the quantification of programmed cell death in murine models and children with neuroblastoma undergoing chemotherapy (10). The observed decreases in choline seen on ${ }^{1} \mathrm{H}$-MRS were not well understood until the group of Clemens and Morley described the inverse relationship of protein translation and apoptosis $(11,12)$. This group found that with the initiation of apoptosis there is an abrupt halt to global protein synthesis in the endoplasmic reticulum as shown in Figure 1, including that of choline and choline-containing lipids and other compounds. The events behind the sudden shutdown of global protein translation are biochemically complex but can be narrowed to several key events, namely inhibition of elongation (or initiation) factor 2 , proteolysis of initiation factors 4GI and 4GII, activation of endoplasmic reticulum transmembrane stress sensors, and inactivation of the progrowth mammalian-target-of-rapamycin pathway.

The interruption of global protein synthesis in the endoplasmic reticulum causes the decrease in choline resonance observed on ${ }^{1} \mathrm{H}-\mathrm{MRS}$ both clinically (13-18) and in animal models $(3,5,19,20) .{ }^{1} \mathrm{H}-\mathrm{MRS}$ has also been successful in the brain, breast, lower extremities, and liver using clinical 1.5or 3.0-T MRI units and coils. Paradoxically, increases in total choline have also been observed in tumors treated with pan-protein synthesis inhibitors of heat-shock protein 90 or histone deacetylase (new selective anticancer drugs with noncytotoxic mechanisms of action) (21). The rise in total choline with these drugs may be related to the induction of autophagy (formation of numerous double-membrane vesicles in the cytoplasm) as opposed to apoptosis.

\section{DWI of Solid Tumors Before and After Therapy}

DWI may also be a useful biomarker of therapeutic efficacy in patients with cancer $(17,22-24)$. DWI relies on the spin labeling of water molecules with a strong electromagnetic pulse and then the following of the spin-labeled water molecules over a short time (i.e., $50 \mathrm{~ms}$ ). DWI then indirectly measures the distance traveled by these water molecules (usually on the order of $30 \mu \mathrm{m}$ ) in this short time. On average, water molecules that are restricted by their local microenvironment (inside a cell as opposed to the extracellular space) travel or diffuse less than water present in regions of necrosis, inflammation, edema, or cysts. The average distances traveled by water molecules in a given voxel are referred to as the average diffusion coefficient (ADC).

Tumors in general have higher cellular density and therefore lower ADCs than do normal tissues and benign tumors (25). With successful therapy, these low ADCs rapidly increase over the course of several days and appear to correspond to tumor cell loss and expansion of the extracellular space in a tumor as shown in Figure $1(26,27)$. Therapeutic efficacy of the apoptosis-induction strategy has been detected as early as $3 \mathrm{~d}$ after dosing, before changes in tumor volume occur (28). The mean ADC increase in tumors was linearly proportional to the mean apoptotic cell density and was inversely proportional to the mean proliferating cell density.

Although DWI holds promise as a marker of treatment efficacy, the changes in ADC are relatively small (usually 
$<50 \%$ of baseline values) and can be affected by inflammation, blood flow, cardiac and respiratory motion, and the presence of necrosis. DWI will also need to be standardized in terms of tumor type, specific therapy, and other acquisition parameters before it can come into widespread clinical use in oncology.

\section{High-Frequency Ultrasound, Optical Coherence Tomography, and Fourier Transform Mid-Infrared Spectromicroscopy}

High-frequency ultrasound (10 $\mathrm{MHz}$ or greater) has been used to detect the unique specular reflections of apoptotic cells in vitro and in vivo (29-31). These specular reflections arise predominately from the peripheral condensation (clumping) and fragmentation of the cell nucleus and DNA (i.e., DNA laddering) in the latter phases of apoptosis as shown in Figure 1. Transducers operating at 10-60 MHz generate ultrasound wavelengths of 25-150 $\mu \mathrm{m}$, which approach the size of individual cells and nuclei (10-20 $\mu \mathrm{m})$ and are therefore sensitive to changes in cell size and nuclear morphology that occur with apoptosis (32). In fact, backscatter from apoptotic nuclei is up to 6-fold greater than that from nonapoptotic cellular nuclei and occurs in cultures treated with a variety of drugs and radiation.

The main limitation of high-frequency ultrasound is that in soft tissue the beam has poor penetration, ranging from 2 to $5 \mathrm{~cm}$ for $10-$ to $30-\mathrm{MHz}$ ultrasound transducers. This inherent limitation can partly be overcome by applying highfrequency ultrasound backscatter analyses to relatively superficial tumors of the skin and breast or by using endoscopic probes for nasopharyngeal and gastrointestinal cancers.

Optical coherence tomography is another form of backscatter spectroscopy and, as opposed to ultrasound, uses laser light with a central wavelength of $1,325 \mathrm{~nm}$ and a $-3-\mathrm{dB}$ bandwidth of approximately $100 \mathrm{~nm}$ with an axial resolution of $9 \mu \mathrm{m}(33,34)$. As with ultrasound, there are a variety of microscopic scattering and reflective interfaces within a cell that arise with apoptosis. Optical coherence tomography identifies increased backscatter of laser light from cells undergoing apoptosis and mitotic arrest, whereas cells undergoing necrosis have a decrease. These changes are linked to structural changes seen on histologic examination of cell samples. These results indicate that optical coherence tomography-integrated backscatter and first-order envelope statistics can be used to detect, and potentially differentiate, modes of cell death in vitro. Clinical application, however, will be limited to endoscopic or ophthalmologic applications because of the poor soft-tissue penetration of light that has the wavelengths used for optical coherence tomography.

Mid-infrared region spectroscopy $(\sim 2.5$ - to $15.5-\mu \mathrm{m}$ wavelength, or $\sim 4,000-650 \mathrm{~cm}^{-1}$ wave number) is another nondestructive microscopic imaging technique (axial resolution of $<1 \mu \mathrm{m}$ ) that generates fingerprintlike spectra of the characteristic vibrational frequencies of various chemical bonds and, therefore, functional groups (35). The high real-time sensitivity of mid-infrared spectroscopy is due to cellular water that is juxtaposed and interacting with ions or biomolecules of interest, thereby structuring water molecules. Structured (as opposed to free) water molecules form distinctive vibrational patterns that can be detected by the absorption patterns of incident mid-infrared region light spectroscopy and constitutes a major advantage of infrared spectromicroscopy over other vibrational methods such as Raman spectroscopy. Fourier transform mid-infrared spectromicroscopy allows the study of individual living cells with a high signal-to-noise ratio, exploiting the continuous, intense, and nondestructive mid-infrared light spectrum available from a cyclotron $(36,37)$. Spectral changes with apoptosis include a shift in the protein amide I and II bands (changing protein morphologies and oxidative state) and a significant increase in the ester carbonyl $\mathrm{C}=\mathrm{O}$ peak, at $1,743 \mathrm{~cm}^{-1}$. For tissues with intrinsic refractive (reflective) interfaces such as seen in atherosclerotic vessels or the retina, it is possible to characterize the features of apoptosis and other forms of cell death (38).

\section{CONCLUSION}

In the short term, ${ }^{1} \mathrm{H}$-MRS imaging of changes in choline signal with therapy appears to be the most direct path for imaging of apoptosis in response to therapy, even in regions and organs outside the brain, including the breast, liver, extremities, and cervix. Decreases in choline directly reflect the silencing of protein translation induced by apoptosis and autophagy. In addition, ${ }^{1} \mathrm{H}-\mathrm{MRS}$ can also readily be performed with existing clinical MRI units, coils, and software without the need for an intravenous contrast agent, making this method attractive for assessing therapeutic response in clinical drug trials, especially in the neoadjuvant setting.

\section{DISCLOSURE}

No potential conflict of interest relevant to this article was reported.

\section{REFERENCES}

1. Blankenberg FG, Strauss HW. Recent advances in the molecular imaging of programmed cell death: part I-pathophysiology and radiotracers. J Nucl Med. 2012;53:1659-1662.

2. Lee SC, Poptani H, Pickup S, et al. Early detection of radiation therapy response in non-Hodgkin's lymphoma xenografts by in vivo ${ }^{1} \mathrm{H}$ magnetic resonance spectroscopy and imaging. NMR Biomed. 2010;23:624-632.

3. Delikatny EJ, Chawla S, Leung DJ, Poptani H. MR-visible lipids and the tumor microenvironment. NMR Biomed. 2011;24:592-611.

4. Zietkowski D, Payne GS, Nagy E, Mobberley MA, Ryder TA, deSouza NM. Comparison of NMR lipid profiles in mitotic arrest and apoptosis as indicators of paclitaxel resistance in cervical cell lines. Magn Reson Med. 2012;68:369377.

5. Liimatainen T, Hakumaki JM, Kauppinen RA, Ala-Korpela M. Monitoring of gliomas in vivo by diffusion MRI and ${ }^{1} \mathrm{H}$ MRS during gene therapy-induced apoptosis: interrelationships between water diffusion and mobile lipids. NMR Biomed. 2009;22:272-279.

6. Musacchio T, Toniutti M, Kautz R, Torchilin VP. ${ }^{1} \mathrm{H}$ NMR detection of mobile lipids as a marker for apoptosis: the case of anticancer drug-loaded liposomes and polymeric micelles. Mol Pharm. 2009;6:1876-1882. 
7. Lyng H, Sitter B, Bathen TF, et al. Metabolic mapping by use of high-resolution magic angle spinning ${ }^{1} \mathrm{H}$ MR spectroscopy predicts apoptosis in cervical carcinomas. BMC Cancer. 2007;7:11.

8. De Silva SS, Payne GS, Morgan VA, et al. Epithelial and stromal metabolite changes in the transition from cervical intraepithelial neoplasia to cervical cancer: an in vivo ${ }^{1} \mathrm{H}$ magnetic resonance spectroscopic imaging study with ex vivo correlation. Eur Radiol. 2009;19:2041-2048.

9. Le HC, Lupu M, Kotedia K, Rosen N, Solit D, Koutcher JA. Proton MRS detects metabolic changes in hormone sensitive and resistant human prostate cancer models CWR22 and CWR22r. Magn Reson Med. 2009;62:1112-1119.

10. Lindskog M. Proton magnetic resonance spectroscopy in neuroblastoma: current status, prospects and limitations. Cancer Lett. 2005;228:247-255.

11. Clemens MJ, Bushel M, Jeffrey IW, Pain VM, Morley SJ. Translation initiation factor modifications and the regulation of protein synthesis in apoptotic cells. Cell Death Differ. 2000;7:603-615.

12. Morley SJ, Coldwell MJ, Clemens MJ. Initiation factor modifications in the preapoptotic phase. Cell Death Differ. 2005;12:571-584.

13. Sharma U, Baek HM, Su MY, Jagannathan NR. In vivo ${ }^{1} \mathrm{H}$ MRS in the assessment of the therapeutic response of breast cancer patients. NMR Biomed. 2011;24:700-711.

14. Bonekamp S, Shen J, Salibi N, Lai HC, Geschwind J, Kamel IR. Early response of hepatic malignancies to locoregional therapy: value of diffusion-weighted magnetic resonance imaging and proton magnetic resonance spectroscopy. J Comput Assist Tomogr. 2011;35:167-173.

15. Hsieh T-J, Li CW, Chuang HY, Liu GC, Wang CK. Longitudinally monitoring chemotherapy effect of malignant musculoskeletal tumors with in vivo proton magnetic resonance spectroscopy: an initial experience. J Comput Assist Tomogr. 2008;32:987-994.

16. Tozaki M, Sakamoto M, Oyama Y, et al. Monitoring of early response to neoadjuvant chemotherapy in breast cancer with ${ }^{1} \mathrm{H}$ MR spectroscopy: comparison to sequential $2-\left[{ }^{18} \mathrm{~F}\right]-$ fluorodeoxyglucose positron emission tomography. J Magn Reson Imaging. 2008;28:420-427.

17. Tozaki M, Oyama Y, Fukuma E. Preliminary study of early response to neoadjuvant chemotherapy after the first cycle in breast cancer: comparison of ${ }^{1} \mathrm{H}$ magnetic resonance spectroscopy with diffusion magnetic resonance imaging. Jpn J Radiol. 2010;28:101-109.

18. Tozaki M, Sakamoto M, Oyama Y, Maruyama K, Fukuma E. Predicting pathological response to neoadjuvant chemotherapy in breast cancer with quantitative ${ }^{1} \mathrm{H}$ MR spectroscopy using the external standard method. J Magn Reson Imaging. 2010;31:895-902.

19. Jensen LR, Huuse EM, Bathen TF, et al. Assessment of early docetaxel response in an experimental model of human breast cancer using DCE-MRI, ex vivo HR MAS, and in vivo ${ }^{1} \mathrm{H}$ MRS. NMR Biomed. 2010;23:56-65.

20. Huuse EM, Jensen LR, Goa PE, et al. Monitoring the effect of docetaxel treatment in MCF7 xenografts using multimodal in vivo and ex vivo magnetic resonance methods, histopathology, and gene expression. Transl Oncol. 2010; 3:252-263.

21. Podo F, Canevari S, Canese R, Pisanu ME, Ricci A, Iorio E. MR evaluation of response to targeted treatment in cancer cells. NMR Biomed. 2011;24:648-672.

22. Radermacher KA, Magat J, Bouzin C, et al. Multimodal assessment of early tumor response to chemotherapy: comparison between diffusion-weighted MRI,
${ }^{1} \mathrm{H}-\mathrm{MR}$ spectroscopy of choline and USPIO particles targeted at cell death. NMR Biomed. 2012;25:514-522.

23. Wybranski C, Zeilxe M, Löwenthal D, et al. Value of diffusion weighted MR imaging as an early surrogate parameter for evaluation of tumor response to high-dose-rate brachytherapy of colorectal liver metastases. Radiat Oncol. $2011 ; 6: 43$.

24. Dudeck O, Zeile M, Wybranski C, et al. Early prediction of anticancer effects with diffusion-weighted MR imaging in patients with colorectal liver metastases following selective internal radiotherapy. Eur Radiol. 2010;20:2699-2706.

25. Padhani AR, Liu G, Koh DM, et al. Diffusion-weighted magnetic resonance imaging as a cancer biomarker: consensus and recommendations. Neoplasia. 2009;11:102-125.

26. Rozel S, Galbán CJ, Nicolay K, et al. Synergy between anti-CCL2 and docetaxel as determined by DW-MRI in a metastatic bone cancer model. $J$ Cell Biochem. 2009; 107:58-64.

27. Huang MQ, Pickup S, Nelson DS, et al. Monitoring response to chemotherapy of non-Hodgkin's lymphoma xenografts by T2-weighted and diffusion-weighted MRI. NMR Biomed. 2008;21:1021-1029.

28. Kim H, Morgan DE, Zeng H, et al. Breast tumor xenografts: diffusion-weighted MR imaging to assess early therapy with novel apoptosis-inducing anti-DR5 antibody. Radiology. 2008;248:844-851.

29. Vlad RM, Kolios MC, Moseley JL, Czarnota GJ, Brock KK. Evaluating the extent of cell death in $3 \mathrm{D}$ high frequency ultrasound by registration with whole-mount tumor histopathology. Med Phys. 2010;37:4288-4297.

30. Banihashemi B, et al. Ultrasound imaging of apoptosis in tumor response: novel preclinical monitoring of photodynamic therapy effects. Cancer Res. 2008;68: 8590-8596.

31. Vlad RM, Alajez NM, Giles A, Kolios MC, Czarnota GJ. Quantitative ultrasound characterization of cancer radiotherapy effects in vitro. Int J Radiat Oncol Biol Phys. 2008;72:1236-1243.

32. Vlad RM, Kolios MC, Czarnota GJ. Ultrasound imaging of apoptosis: spectroscopic detection of DNA-damage effects at high and low frequencies. Methods Mol Biol. 2011;682:165-187.

33. Farhat G, et al. Detecting cell death with optical coherence tomography and envelope statistics. J Biomed Opt. 2011;16:026017.

34. Strohm E, Czarnota GJ, Kolios MC. Quantitative measurements of apoptotic cell properties using acoustic microscopy. IEEE Trans Ultrason Ferroelectr Freq Control. 2010;57:2293-2304.

35. Holman HY, Martin MC, Blakely EA, Bjornstad K, McKinney WR. IR spectroscopic characteristics of cell cycle and cell death probed by synchrotron radiation based Fourier transform IR spectromicroscopy. Biopolymers. 2000; 57:329-335.

36. Holman HY, Bechtel HA, Hao Z, Martin MC, Synchrotron IR. Spectromicroscopy: chemistry of living cells. Anal Chem. 2010;82:8757-8765.

37. Chen L, Holman HY, Hao Z, et al. Synchrotron infrared measurements of protein phosphorylation in living single PC12 cells during neuronal differentiation. Anal Chem. 2012;84:4118-4125.

38. Holman HY, Bjornstad KA, Martin MC, McKinney WR, Blakely EA, Blankenberg FG. Mid-infrared reflectivity of experimental atheromas. J Biomed Opt. 2008;13:030503. 